\title{
PREVALENCIA Y FACTORES ASOCIADOS A DISFUNCIÓN AUDITIVA EN PREMATUROS DE MUY BAJO PESO AL NACER EN EL INMP. 2009-2011
}

\author{
María Mercedes Morillo Acuña ${ }^{1}$, Tania Guisella Paredes Quiliche²
}

\begin{abstract}
RESUMEN:
Objetivos. Determinar la prevalencia y los principales factores asociados a disfunción auditiva en prematuros de muy bajo peso al nacer atendidos en el INMP desde Octubre 2009 a diciembre 2010. Materiales y métodos. Estudio analítico prospectivo. Incluyó a todos los prematuros de muy bajo peso al nacer ingresados al INMP de octubre del 2009 a diciembre 2011. Previa evaluación clínica se aplicaron las pruebas de Emisiones otoacústicas previo al egreso hospitalario o a los 30 días de edad postnatal a los 3 meses de edad corregida, durante el seguimiento. Resultados. Se encontró una prevalencia de Hipoacusia en 9.2/1000 RNMBP; 30 pacientes quedaron con resultado dudoso en el primer examen de EOA debido a la deserción al control. Conclusiones. La prevalencia de disfunción auditiva en prematuros de muy bajo peso al nacer en nuestro medio fue elevada (9.2/1000), coincide con lo referido en la literatura, es necesario implementar un programa de tamizaje auditivo universal en este grupo poblacional del INMP debido a las consecuencias sociales y en el neurodesarrollo.
\end{abstract}

Palabra clave: Recién nacido prematuro; Disfunción auditiva (fuente: DeCS BIREME).

\section{PREVALENCE AND FACTORS ASSOCIATED WITH AUDITORY DYSFUNCTION IN PREMATURES INFANT OF VERY LOW BIRTH WEIGHT IN THE INMP. 2009-2011}

\begin{abstract}
Objectives.To determine the prevalence and the main factors associated with auditory dysfunction in prematures infant of very low weight attended at birth from October 2009 to December 2010. Material and methods. Prospective analytical study. It included all prematures infant of very low weight at birth admitted to the INMP from October 2009 to December 2011. Prior clinical evaluation it applied emissions testing otoacoustic prior to hospital discharge or 30 days of postnatal age 3 months of corrected age, during follow-up. Results. It was found a prevalence of hearing loss in 9.2/1000 RNMBP; 30 patients were with uncertain outcome in the first examination of EOA because of desertion to the control. Conclusions. The prevalence of auditory dysfunction in prematures infant of very low weight at birth in our environment was high (9.2/1000), matches in the literature, it is necessary to perform a programme of universal newborn hearing screening in this population of the INMP due to the consequences social and in the neurodevelopment.
\end{abstract}

Key word: Newborn premature; Auditory dysfunction (source: MeSH NLM).

\section{INTRODUCCION}

Con la implementación tecnológica de las Unidades de Cuidados Intensivos neonatales (UCIN) ocurrida en las ultimas décadas, se ha logrado mejorar la sobrevida de los recién nacidos prematuros especialmente del grupo de muy bajo peso al nacer ( $<1,500$ gramos) y actualmente la sobrevida tiene niveles variables dependiendo del mayor o menor desarrollo tecnológico de los países.

En el Instituto Nacional Materno Perinatal (INMP), en el año 2004 se fortalece el Servicio de Cuidados Intensivos Neonatales con equipamiento de tecnología innovadora y a partir de esa fecha, se produce un cambio en la calidad de la atención y los resultados. El INMP se convierte en un centro de referencia nacional para el cuidado intensivo del recién nacido y de la madre de alto riesgo, logrando progresivamente incrementar la sobrevida de prematuros de muy bajo peso y extremo bajo peso al nacer $(<1,000$ gramos). En el INMP en el año 2007, la sobrevida de prematuros de MBPN fue $85 \%$ y de los RN de extremo bajo peso al nacer (EBPN) de 45\%; y en el año 2011 la sobrevida en el grupo de RN de extremo bajo peso subió a $58 \%$.

Con la mejoría en la sobrevida en el grupo de prematuros extremos, se ha logrado identificar la existencia de un mayor riesgo de discapacidad entre los sobrevivientes; la cual está relacionada con factores biológicos y al grado de intervención a la que estos recién nacidos son sometidos (ventilación mecánica asistida, administración de diuréticos, antibióticos, oxígeno en concentraciones altas, etc.) para lograr reducir la mortalidad y controlar las numerosas complicaciones que presentan durante su evolución1.

Médico Neonatólogo del Departamento de Neonatología del Instituto Nacional Materno Perinatal. Lima Perú.

2 Médico Pediatra Jefe del servicio de Cuidados Intensivos Neonatal del Instituto Nacional Materno Perinatal. Lima- Perú.

Recibido: 10-10-13 Aprobado: 3-12-13 
Los principales tipos de discapacidad se clasifican en disfunción motora, alteración en el desarrollo mental y disfunción sensorial. Según reportes internacionales, la disfunción auditiva en el grupo de prematuros es muy importante, siendo de variable severidad y afectan en forma permanente la calidad de vida de los pacientes ${ }^{2}$. Trabajos sobre neurodesarrollo realizados en México ${ }^{8}$ reportan las secuelas en la audición, lenguaje, y estado de salud general; así como la alteración en el sistema nervioso central por lo que otro aspecto muy importante a tener en cuenta en este grupo de sobrevivientes, es la calidad de vida.

La audición es un factor poco estudiado en nuestro medio de manera sistematizada dentro del periodo de hospitalización en la UCIN, donde se utilizan algunas medidas terapéuticas que pueden ocasionar lesiones auditivas tales como la ventilación mecánica artificial por más de cinco días, utilización de fármacos ototóxicos como aminoglucósidos y diuréticos, hiperbilirrubinemia que requiere recambio sanguíneo, hemorragia intraventricular (HIV), retinopatía de la prematuridad (ROP), infecciones graves (sepsis, meningitis), hipertensión pulmonar persistente y sufrimiento fetal con hipoxia significativa.

La audición es el mecanismo a través del cual se adquiere el lenguaje por ello es necesario la detección oportuna de los casos de hipoacusia y su rehabilitación, desde la etapa neonatal hasta antes de los seis meses de edad postnatal para mejorar las expectativas ${ }^{9,10,11}$. La hipoacusia neurosensorial o disminución de la percepción auditiva es un problema de especial importancia durante la infancia ya que el desarrollo intelectual y social del niño está íntimamente ligado a las aferencias auditivas del sistema nervioso central (SNC), cuyo potencial discapacitante y minusvalidante depende de la precocidad en el diagnostico y de su tratamiento oportuno así como su rehabilitación, ya que cuanto más tarde se diagnostica a lo largo de la etapa pre lingüística ya sea congénita o adquirida, peores serán los resultados al tratamiento de allí que se ha determinado que el momento óptimo para la identificación de la hipoacusia debe ser antes de los seis meses de edad postnatal ${ }^{12,13,14}$.

Según la Academia Americana de Pediatría (AAP), la edad promedio de detección de Hipoacusia neurosensorial, en los lugares donde no existe un Programa de Screening auditivo es de 14 a 30 meses o más tardío. Debido a esta razón, la AAP recomienda la detección universal de la Hipoacusia neurosensorial antes de los 3 meses de vida con intervención apropiada antes de los 6 meses y en EEUU se aplica bajo forma de ley desde el año 2000.

En nuestro país, no solo carecemos de datos que nos aproximen a conocer la magnitud y severidad de la disfunción auditiva en neonatos prematuros; sino que también desconocemos la relación entre las intervenciones o procedimientos terapéuticos, a los que deben ser expuestos durante la hospitalización en las Unidades neonatales, y los resultados auditivos a largo plazo. Esta situación resulta muy preocupante por las implicancias en la calidad de vida de los pacientes, sobretodo cuando la disfunción auditiva no es detectada precozmente, por lo que constituye un problema de salud pública. Es por ello que se hace necesario determinar la prevalencia y los principales factores asociados a disfunción auditiva en prematuros de muy bajo peso al nacer atendidos en el INMP.

\section{MATERIALES Y MÉTODOS}

Se realizó un estudio analítico de tipo prospectivo, en el Servicio de Cuidados Intermedios y en el Consultorio de seguimiento de niños de alto riesgo del Departamento de Neonatología del Instituto Nacional Materno Perinatal. Ingresaron al estudio los recién nacidos de muy bajo peso al nacer ( $R N<1500$ gramos) hospitalizados en el INMP de octubre del 2009 a diciembre del 2010, que egresaron vivos.

La evaluación auditiva fue realizada mediante Emisiones Otacústicas (EOA), para ello el INMP adquirió un equipo de marca Interaccustic- Ecclipse y las pruebas fueron realizadas por un Otorrinolaringólogo entrenado y una fonoaudióloga quienes registraron los resultados en el instrumento de recolección de datos previamente diseñado y también en la historia clínica del recién nacido. Según el protocolo de seguimiento del INMP, la evaluación auditiva para niños de riesgo se realizó en dos fases:

- La primera evaluación: durante su hospitalización cercana al alta y/o a los 30 días de edad postnatal (lo que se presentara primero)

- La segunda evaluación: a los 3 meses en el consultorio de Seguimiento del niño de alto riesgo del INMP.

Los controles de seguimiento fueron realizados hasta fines de marzo del 2011.

Para efectos del análisis estadístico a la población de estudio se les dividió en dos grupos:

- Grupo 1 (casos): RNPT con peso de nacimiento menor de $1500 \mathrm{~g}$ nacidos en el INMP durante el periodo de estudio y con disfunción auditiva confirmada a los 3 meses de edad corregida.

- Grupo 2 (controles): RNPT con peso de nacimiento menor de $1500 \mathrm{~g}$, nacidos en el INMP durante el periodo de estudio y sin disfunción auditiva a los 3 meses de edad corregida.

En ambos grupos, se buscó en forma retrospectiva mediante la revisión de la historia clínica materna y neonatal, la exposición a los factores de riesgo estudiados. El registro de los datos se realizó en el formato de recolección de información, directamente de la 
Criterios de inclusión:

- Recién nacidos prematuros de muy bajo peso al nacer atendidos en el Instituto Nacional Materno Perinatal de octubre del 2009 a diciembre del 2010.

- Recién nacidos prematuros de muy bajo peso al nacer vivos al alta.

- Recién nacidos que se realizaron las EOA y/o potenciales evocados auditivos para confirmación diagnóstica.

- Los que tengan Historia clínica completa.

Criterios de exclusión:

- RN con malformaciones congénitas mayores y/ o múltiples.

- Antecedentes maternos de infección viral tipo TORCHS

- Prematuros que presentan malformación craneofacial

El procesamiento de los datos se realizó utilizando el programa estadístico SPSS 17.0

Se realizó un análisis uní variado, bivariado y multivariado para la determinación de los principales factores asociados a disfunción auditiva.

Se presentan tablas de distribución de frecuencias para efectos de describir las principales características de la población incluida en la investigación. Las variables cuantitativas fueron descritas mediante medidas de tendencia central como promedios y medidas de dispersión.

Se utilizó la prueba de X2 para cada uno de los Factores de Riesgo, considerando como significativo cuando $p<$ 0.05 .

Para factores con diferencia significativa, se calculó el Odds Ratio (OR) con un intervalo de confianza de 95\%.

Para datos cuantitativos se utilizó la prueba de la $t$ de Student para la comparación de medias, considerándose como significativo una $p<$ de 0.05 .

Se utilizó el modelo de Regresión logística, para lo cual se codificó la variable disfunción auditiva de tipo categórico dicotómica y se ingresó al análisis las variables independientes que resultaron significativas en el modelo.

\section{RESULTADOS}

Durante el periodo de estudio (de octubre del 2009 a diciembre 2010) nacieron un total de 21,432 recién nacidos vivos, de los cuales 326 correspondieron a neonatos con peso menor de 1500 gramos al nacimiento, de los cuales fueron evaluados 175 recién nacidos (44 correspondieron al año 2009 y 131 al año 2010). El 38,9\% (68 pacientes) fueron del sexo masculino y $61,1 \%$ (107 pacientes) femenino. La distribución de acuerdo al peso y edad gestacional lo podemos ver en la tabla 1.

Tabla 1. Distribución de RN según peso y edad gestacional al nacimiento. Recién nacidos menores de 1500 gr. 2009 - 2010. Departamento de Neonatología INMP.

\begin{tabular}{lccc}
\hline \multicolumn{1}{c}{ Peso } & No & $\%$ & \% acumulado \\
\hline Menor de $750 \mathrm{~g}$ & 10 & 5,7 & 5,7 \\
750 a $999 \mathrm{~g}$ & 30 & 17,1 & 22,8 \\
\hline 1000 a $1249 \mathrm{~g}$ & 51 & 29,1 & 51,9 \\
1250 a $1500 \mathrm{~g}$ & 84 & 48,1 & 100,0 \\
$\quad$ Edad gestacional & No & $\%$ & $\%$ acumulado \\
Menor de 26 sem & 3 & 1,7 & 1,7 \\
26 a 29 sem & 41 & 23,4 & 25,1 \\
30 a 33 sem & 108 & 61,7 & 86,9 \\
34 a 36 sem & 23 & 13,1 & 100,0 \\
\hline
\end{tabular}

La media del peso de nacimiento fue $1180 \mathrm{~g}$ (DE 222 g), de la edad gestacional 30,9 sem. (DE 2,4 sem.) y del perímetro cefálico fue $26,9 \mathrm{~cm}$ (DE 2,1 cm). El test de Apgar al minuto tuvo como media $6,3(\mathrm{DE} 1,9)$ y a los 5 minutos 7,9 (DE 1,3). Tabla 2.

Tabla 2. RN según puntaje de Apgar al minuto y a los cinco minutos. Recién nacidos menores de 1500 gr. 2009 - 2010. Departamento de Neonatología INMP

\begin{tabular}{cccc}
\hline Puntaje Apgar al minuto & No & $\%$ & $\%$ acumulado \\
\hline 1 a 3 & 24 & 13,7 & 13,7 \\
4 a 6 & 48 & 27,4 & 41,1 \\
\hline 7 a 10 & 103 & 58,9 & 100,0 \\
\hline Apgar a los 5 minutos & No & $\%$ & $\%$ acumulado \\
1 a 3 & 3 & 1,7 & 1,7 \\
4 a 6 & 15 & 8,6 & 10,3 \\
\hline 7 a 10 & 157 & 89,7 & 100,0 \\
\hline
\end{tabular}

De los 175 RN, $163(91,1 \%)$ nacieron en el INMP y 12 $(8,9 \%)$ fueron referidos de otros hospitales.

Se realizaron EOA a $175 \mathrm{RN}$ considerados de riesgo para disfunción auditiva, los resultados fueron normales en 137 niños (78.3\%), dudoso en $30(17.1 \%)$ y anormal en $8(4.6 \%)$. De los 30 pacientes con resultado dudoso en la primera prueba y debido a que no acudieron para realizarles la segunda prueba (deserción al seguimiento en Consultorio) no se pudo completar el diagnóstico. En 24 casos los pacientes tenían resultados de alteración unilateral. En todos los casos se notificó telefónicamente a los familiares, insistiéndose en repetidas oportunidades; incluyendo en algunos casos visitas domiciliares sin lograrse ubicar a las familias en las direcciones registradas. A los pacientes con resultado anormal de EOA se les 
realizó Potenciales evocados auditivos (PEA); de ellos 5 fueron normales y 3 tuvieron hipoacusia neurosensorial, 1 bilateral y 2 de oído izquierdo.

El análisis bivariado mostró que ninguno de los factores prenatales reportados en estudios previos obtuvo un nivel de significancia estadística entre los pacientes con Emisiones otacústicas normales y anormales.

En lo que respecta a los factores de riesgo postnatal antropométricos y condición al nacimiento, ninguno obtuvo un nivel de significancia estadística entre los pacientes con Emisiones otacústicas normales y anormales.

Finalmente, las complicaciones durante la etapa neonatal: meningoencefalitis (MEC), sepsis y convulsiones no resultaron ser estadísticamente significativas; sin embargo el procedimiento de exsanguineotransfusión mostró una elevada significación estadística con disfunción auditiva (Tabla 3).

Tabla 3. Análisis bivariado de factores prenatales, natales y postnatales y su relación con EOA. Recién nacidos menores de 1500 gr. 2009 - 2010. Departamento de Neonatología INMP

\begin{tabular}{lccccc}
\hline \multirow{2}{*}{ Factor prenatal } & \multicolumn{2}{c}{$\begin{array}{c}\text { EOA } \\
\text { anormales }\end{array}$} & \multicolumn{2}{c}{$\begin{array}{c}\text { normales } \\
\text { no }\end{array}$} & P \\
\cline { 2 - 5 } & No & $\%$ & No & $\%$ & \\
\hline No corticoides & 3 & 37,5 & 51 & 31,9 & 0.7 \\
Diabetes & 0 & 0 & 2 & 1,3 & 0.9 \\
TORCHS & 1 & 12,5 & 1 & 0,6 & 0,09 \\
Uso de Antibacterianos & 4 & 50 & 84 & 50,3 & 0,85 \\
Antecedente familiar & 0 & 0 & 1 & 0,7 & 0.9 \\
Factor natales & & & & & \\
Peso menor de 1000 gramos & 2 & 25 & 38 & 22,8 & 0,58 \\
EG menor de 29 semanas & 1 & 12,5 & 24 & 14,4 & 0,68 \\
Sexo masculino & 4 & 50 & 64 & 38,3 & 0,37 \\
Apgar bajo al minuto & 3 & 37,5 & 69 & 41,3 & 0,56 \\
Apgar bajo a los 5 minutos & 1 & 12,5 & 17 & 10,2 & 0,58 \\
Factor postnatales & & & & & \\
Sepsis (hemocultivo + ) & 0 & 0 & 32 & 19,3 & 0,19 \\
MEC & 0 & 0 & 5 & 3,0 & 0,78 \\
Convulsión & 0 & 0 & 3 & 1,8 & 0,86 \\
ETT & 2 & 25,0 & 3 & 1,8 & 0,01 \\
Furosemida & 0 & 0 & 52 & 31,2 & 0,08 \\
ATB & 8 & 100 & 153 & 91,6 & 0,5 \\
\hline
\end{tabular}

La media de la Bilirrubina total en el grupo de EOA anormal y el grupo normal fue de 8,5 y 8 respectivamente, no encontrándose diferencia significativa; siendo la indicación de exsanguineotransfusión basada en el peso de nacimiento, la edad del paciente en el momento de mayor nivel de bilirrubinas y otras complicaciones que incrementan el riesgo de impregnación de los ganglios basales por este metabolito (estado de shock, hipoproteinemia, acidosis metabólica, etc).

El promedio de días de ventilación mecánica fue de 4 para el primer grupo y 7 para el segundo, sin encontrarse diferencia significativa.

\section{DISCUSIÓN}

En los últimos años el avance en el conocimiento de la fisiología del feto y del neonato así como de la fisiopatología de las enfermedades más frecuentes; sumado al desarrollo de la tecnología en el diagnostico y tratamiento, han hecho posible el aumento de la supervivencia de los RNMBP. Pese a la asistencia prenatal para llevar al feto a un estado de madurez para su adaptación normal al nacimiento, y lograr a obtener el máximo desarrollo de sus potencialidades, esta meta se ve amenazada cuando el nacimiento se produce antes de alcanzar el término de la gestación cuyo resultado es el parto prematuro con peso debajo de lo normal constituyendo un grupo de alto riesgo de morbimortalidad con patologías como hemorragia intraventricular (HIV), Retinopatía de la prematuridad (ROP), displasia broncopulmonar (DBP), alteraciones del neurodesarrollo graves o sutiles que han producido un aumento de las secuelas discapacitantes como los reporta la literatura internacional entre ellas la discapacidad auditiva. El peso es la variable antropométrica de mayor uso en la evaluación del recién nacido y sirve para establecer las pautas de manejo y pronostico, no solo para el periodo neonatal sino para toda la vida ${ }^{1-6}$.

La denominación de recién nacido de muy bajo peso (RNMBP), que se refiere a los RN con peso menor a 1500 gramos e implica trabajar solo con la variable peso de nacimiento, sin tener en cuenta la edad gestacional ${ }^{7}$. Trabajos sobre neurodesarrollo realizados en México reportan las secuelas en la audición, lenguaje, y estado de salud general; así como la alteración en el sistema nervioso central otro aspecto muy importante en este grupo de sobrevivientes, la calidad de vida ${ }^{8}$.

El Comité Conjunto de Audición infantil, en el año 2007, respalda la detección temprana, la intervención de niños con pérdida auditiva y recomienda el uso de Emisiones otoacústicas y Potenciales evocados auditivos de tallo cerebral para tamizaje auditivo ${ }^{14,16}$. Las emisiones otacústicas son un medio de evaluación para la detección de disfunción auditiva, El equipo consta básicamente de un generador de frecuencia (estimulador), una sonda (probeta), un amplificador, un promediador y un analizador de espectro frecuencial.

Su evaluación se ha utilizado como método de tamizaje para identificar la hipoacusia en el neonato, tiene una sensibilidad de $91 \%$ y especificidad de $85 \%$ cuando hay falsos positivos se complementa con los potenciales evocados auditivos con lo cual la sensibilidad aumenta a $100 \%$ y la especificidad aumenta a $98 \%$. Las Emisiones Otacústicas constituyen un método eficaz y confiable para detectar trastornos auditivos en el recién nacido ${ }^{19}$.

La audición es un factor poco estudiado en nuestro medio de manera sistematizada dentro del periodo de hospitalización en UCIN, donde se utilizan algunas medidas terapéuticas 
que pueden ocasionar lesiones auditivas: ventilación mecánica artificial por más de cinco días, uso de fármacos ototóxicos (aminoglucosidos, furosemida), hiperbilirrubinemia que requiere recambio sanguíneo total, morbilidades: Hemorragia intraventricular (HIV), infecciones graves (sepsis, meningitis), la hipoxia significativa y sufrimiento fetal y la hipertensión pulmonar persistente.

Sin embargo en el presente estudio, el análisis bivariado mostró que ninguno de los factores prenatales reportados en estudios previos obtuvo un nivel de significancia estadística entre los pacientes con Emisiones otacústicas normales y anormales excepto la relacionada con hiperbilirrubinemia que requirió exanguinotransfusion. Del mismo modo en lo que respecta a los factores de riesgo postnatal antropométricos y condición al nacimiento, ninguno obtuvo un nivel de significancia estadística entre los pacientes con Emisiones otacústicas normales y anormales como los reportados en la literatura internacional y por el Comité Conjunto para la Audición Infantil (1994, 2007).

La incidencia de hipoacusia o sordera neurosensorial es variable según distintas investigaciones y centros, pueden ir desde 0.5-1/1000 nacidos vivos hasta 1-3/1000 nacidos vivos; esto considerando el total de neonatos. En EEUU los programas de tamizaje tienen una existencia de 20 años aproximadamente, demostrando que entre 2,5 a $10 \%$ de niños de alto riesgo cursan con pérdida auditiva.

La literatura internacional reporta diferentes porcentajes de pacientes con hipoacusia neurosensorial: en el Servicio de otorrinolaringología del Hospital Dr. Sotero del Río en la ciudad de Chile, el $22.8 \%$ de los menores evaluados en neonatología con emisiones otoacústicas son derivados de un segundo tamizaje y $6 \%$ fueron a estudio audiológico para diagnostico definitivo ${ }^{2}$. Un reporte de seguimiento en el Instituto Perinatal de México DF reporta de 134 niños un $20 \%$ presentaron alguna alteración auditiva relacionados cada vez mas frecuente a fármacos ototóxicos, lo que hizo que revisaran las normas en UCIN en cuanto a las dosis, vía de administración, y tiempo de infusión, y el uso alternativo de otros antibióticos para prevenir las lesiones auditivas $^{8}$. Un estudio realizado en el Hospital Universitario de Canarias - España ${ }^{20}$, sobre correlación entre emisiones otacústicas y Potenciales evocados de Tronco encefálico (PEATC), de los 50 niños estudiados, 23 pertenecían a la población de riesgo (46\%), mientras que $27(54 \%)$ de RN no tenían antecedentes familiares de hipoacusia neurosensorial, ni estaban incluidos en los grupos de riesgo que necesitan realizar examen auditivo. De este total de 50 niños, 42 (84\%) presentaban ausencia de otoemisiones acústicas transitorias (OEAT) unilateralmente y en 8 niños (16\%), el fallo fue bilateral. De los 42 bebés que presentaban pérdida unilateral de OEAT en 27 (64\%) se observó ausencia de potenciales evocados auditivos de tronco cerebral en el oído problema con una estimulación de $75 \mathrm{~dB} \mathrm{nHL}$ (PETC) y de los 8 que tenían fallo de OEAT bilateralmente se encontró ausencia de registro de PETC en los 8 (100\%). Cinco de estos ocho niños (62\%) con fallo bilateral, pertenecían a la población de riesgo y/o presentaban antecedentes familiares de hipoacusia neurosensorial. Un resultado muy importante evidenciado en este estudio fue que aproximadamente el $50 \%$ de los niños examinados que no pasaron el tamizaje auditivo no pertenecían a la población de riesgo ni tenían antecedentes de hipoacusia neurosensorial familiar, hecho que justifica la necesidad de incluir en la evaluación auditiva a todo recién nacido vivo ${ }^{20}$. En un estudio prospectivo en recién nacidos internados en neonatología del Hospital Riviera Rossell en Uruguay en el 2001, de 371 neonatos con peso de 835 a 4195 gramos al nacer, encontraron que $8 \%$ de la población tenían factor de riesgo para hipoacusia, cifra similar a las descritas por el Comité Conjunto para la Audición. ${ }^{13}$ Otro estudio realizado en Venezuela en el 2005 en el Hospital del Niño "J. M. de los Ríos" de 31 recién nacidos a término encontraron al $87 \%$ con alteración auditiva: $8 \%$ con afección unilateral y $92 \%$ con afectación bilateral. Los factores de riesgo importantes fueron el uso de fármacos ototoxicos $45 \%$, sepsis y meningitis $25 \%$ otras causas $32 \%{ }^{21}$ En el Hospital Universitario de Valladolid España, de 1277 recién nacidos tamizados 94\% fueron evaluados, $3.3 \%$ presentaba algún factor de riesgo para hipoacusia, el $90 \%$ pasó el examen, $2.5 \%$ paso a evaluación diagnostica. Detectaron precozmente $5.8 / 1000$ nacidos vivos con hipoacusia severa o profunda bilateral. En el Hospital Universitario de San Carlos de Madrid, de 1532 recién nacidos, $0.39 \%$ presentaron hipoacusia permanente con umbral de profundidad superior a $40 \mathrm{~dB}$, prevalencia de hipoacusia bilateral permanente con umbral mayor de $40 \mathrm{~dB}$ fue $2.6 \%$ de recién nacidos ${ }^{22}$

En la investigación realizada en el INMP, se encontraron pruebas de Emisiones otoacústicas anormales en $4.6 \%$ del total de recién nacidos de muy bajo peso evaluados; pero con un $17.1 \%$ adicional de resultados dudosos al no haber completado la segunda evaluación por deserción de los pacientes al seguimiento en el INMP.

Si se consideran solo los casos confirmados de Hipoacusia (mediante la aplicación de la prueba de Potenciales auditivos) la prevalencia de Disfuncion auditiva en este grupo de pacientes de muy bajo peso al nacer en nuestra institución es de 9.2/1,000 RN menores de 1500 gramos similar a los reportados en la literatura internacional, y se debe considerar que esta cifra puede ser aún mayor si se incluye a algunos pacientes que quedaron con resultado final dudoso por deserción.

El efecto de la pérdida auditiva uni o bilateral sobre el desarrollo de la comunicación, el desarrollo cognitivo, conductual y socio emocional así como el desempeño académico y las oportunidades vocacionales y de trabajo, se harán evidentes en los años posteriores y serán catastróficos, por lo que el diagnóstico precoz y la implementación de medidas correctivas y de rehabilitación aplicadas antes de los 6 meses de edad permitirán lograr un mejor desarrollo y calidad de vida para este grupo de pacientes de alta vulnerabilidad. 


\section{CONCLUSIONES}

La prevalencia de disfunción auditiva en prematuros de muy bajo peso al nacer en el INMP fue de 9,2/1000.

El factor de riesgo postnatal asociado fue la hiperbilirrubinemia que requirió exanguineo transfusión total.

\section{RECOMENDACIONES}

Se debe ampliar el tamaño de la muestra para comparar los resultados de los factores postanatales asociados a disfunción auditiva con otros estudios.

Es necesario implementar un programa de tamizaje universal auditivo para detectar precozmente la disfunción auditiva y su intervención oportuna, ya que es un problema de salud pública y de relevancia social.

\section{AGRADECIMIENTO}

A las madres de los pacientes que confiaron en el estudio. Al los directivos del INMP por su apoyo en el desarrollo de la investigación dentro de la misión y visión institucional.

\section{REFERENCIAS BIBLIOGRÁFICAS}

1 Del Moral T, Bancalari E. Cambios en la mortalidad de los prematuros de muy bajo peso. Temas de Pediatría. Asociación Mexicana de Pediatría. Neonatología Ed. Interamericana México 1996: 5-12.

2 Godoy C, Bustamante L. "Evaluación de la fase de screening auditivo en menores con factores de riesgo". Revista Otorrinolaringología y Cirugía de Cabeza Cuello 2006; Vol 66: 103-106.

3 Hernández J, Villanueva D. "El Prematuro y sus problemas". Ginecología y Obstetricia. Editores Ludmir A. Cervantes R Castellanos C.CONCYTEC 1996: 566-583.

4 Denegri J. Mortalidad Perinatal. Ginecología y Obstetricia. Editores Ludmir A. Cervantes R. Castellanos C. CONCYTEC 1996:626-635.

5 Morales, Victor y col "Curvas estándares de peso al nacimiento para neonatos del Paraguay". Archivos Argentinos de Pediatría; 98 (6): 2000, 376-381.

6 Guayasamín, O.; Benedetti W. L; Althabe O. Nieto F. Tenzer Z. "Crecimiento fetal humano valorado por indicadores antropométricos". Boletín de la Oficina Sanitaria Panamericana 1976, 88(6): pág. 481.488

7 Tapia J. Cifuentes J. Ventura Juncá P. El recién nacido con peso inferior a 1500 gramos. Editorial Mediterráneo. Segunda Edición 2000 pág. 80-85

8 Fernández Carrocera, Luis y col. "El neurodesarrollo a los dos años de vida de neonatos tratados en una unidad de cuidados intensivos neonatales" Revista Panamericana de Salud Publica 5(1) 1999, pág. 29-35.

9 Martin, Richard; Fanaroff, Avroy. Neonatal, Perinatal Medicine: Practical, evidence-based advice on perinatology and neonatology. $9^{\text {th }}$ edition. 2010. Mosby

10 Volpe, Joseph. Neurology of the Newborn. $4^{\text {th }}$ edition, año 2000, pag. 531-540

11 Hernandez Herrera R, Hernández Aguirre Ly col. "Parámetros de normalidad de las otoemisiones acústicas en neonatos". Rev. Med .Inst. Mex. Seguro Social 2007; 45 (1): 63-67.

12 Paparella Michael. Tratado de Otorrinolaringología. Tomo II. Editorial Panamericana. Buenos Aires, 1993

13 Ferreira R,Basile L. "Emisiones otoacusticas en recién nacidos con factores de riesgo auditivo" Archivos de Pediatría de Uruguay Vol. 74 (3) pág. 197- 202. 2003.

14 Joint Committee on Infant Hearing. Year 2007 Position Statement: Principles and guidelines for Early Hearing Detection and Intervention Programs. Pediatrics; 120 (4): 898-921. Oct. 2007

15 Cuñarro, Alonso. "Protocolo detección precoz de hipoacusia en recién nacidos" Hospital de Alcorcón. España 11 de Enero 2008

16 Kountakis S, Psifidis A. "Risk factors associated with hearing loss in neonates" American Journal of Otolaryngology Vol. 18 (2) march- April 1997 pág. 90-93.

17 Kennedy, Colin. "Neonatal screening for hearing impairment" Arch. Dis. Child. 2000, 83: 377-383.

18 Brunton, Laurence; Lazo, John. Goodman and Gilman: Las bases farmacológicas de la terapéutica. Editorial Mc Grawn. 11a. Edicion 2006.

19 Mata, J; Rando, I; Shepherd, M. "Importancia de la impedanciometría dentro de un programa de screening auditivo con otoemisiones en niños". Anales de Otorrinolaringología Mexicana 2001 Vol 52 Nro.2: 96-100

20 Campos, M; Lopez Campos, D. "Correlación entre emisiones otoacusticas y PETC. La importancia del empleo combinado" Acta Otorrinolaringología Esp. 2003; 54: 667-670

21 Fuguet, María; Herize, Mariangel "Hipoacusia en recién nacidos con factores de riesgo perinatal" Archivos Venezolanos de Puericultura y pediatría, 2006 Vol. 69(4), 137-141.

22 La Torre A, Tapia T. "Protocolo combinado de cribado auditivo universal” Anales Españoles de Pediatría 2002; Vol. 57 (1) pág. 55-59.

\section{ORCID iDs}

María Mercedes Morillo Acuña

Tania Paredes Quiliche https://orcid. org/0000-0003-0985-4849

https://orcid.org/0000-0003-3426-4250 